\title{
Effective range from tetramer-dissociation data for cesium atoms
}

\author{
M. R. Hadizadeh, ${ }^{1}$ M. T. Yamashita, ${ }^{1}$ Lauro Tomio,,${ }^{1,2}$ A. Delfino, ${ }^{3}$ and T. Frederico ${ }^{4}$ \\ ${ }^{1}$ Instituto de Física Teórica, Universidade Estadual Paulista, 01140-070, São Paulo, SP, Brazil \\ ${ }^{2}$ Universidade Federal do ABC, 09210-170, Santo André, SP, Brazil \\ ${ }^{3}$ Instituto de Física, Universidade Federal Fluminense, 24210-346, Niterói, RJ, Brazil \\ ${ }^{4}$ Instituto Tecnológico de Aeronáutica, DCTA, 12228-900, São José dos Campos, SP, Brazil
}

(Received 6 November 2012; published 17 January 2013)

\begin{abstract}
The shifts in the four-body recombination peaks, due to an effective range correction to the zero-range model close to the unitary limit, are obtained and used to extract the corresponding effective range of a given atomic system. The approach is applied to an ultracold gas of cesium atoms close to broad Feshbach resonances, where deviations of experimental values from universal model predictions are associated with effective range corrections. The effective range correction is extracted with a weighted average given by $3.9 \pm 0.8 R_{\mathrm{vdW}}$, where $R_{\mathrm{vdW}}$ is the van der Waals length scale, which is consistent with the van der Waals potential tail for the $\mathrm{Cs}_{2}$ system. The method can be generally applied to other cold atom experimental setups to determine the contribution of the effective range to the tetramer dissociation position.
\end{abstract}

DOI: 10.1103/PhysRevA.87.013620

PACS number(s): 67.85.-d, 03.65.Ge, 05.10.Cc, 21.45.-v

\section{INTRODUCTION}

The fundamental quantum properties of atomic systems are under intense investigation in recent cold-atom experiments, in which long-time predicted universal aspects of few-body physics can be probed. In this regard, the increasing number of three-body bound states (trimers), emerging when the absolute value of the two-body scattering length is moved to infinity, known as the Efimov effect [1], was confirmed in different cold-atom laboratories [2-5].

Actually, by extending to more than three particles the search for fundamental quantum behavior in the realm of four-body physics, it is of interest to test the positions of four-atom resonant recombination peaks [6-9], which appear as the two-body scattering length is tuned close to an $s$-wave Feshbach resonance (see, e.g., [10]).

On the theoretical side, the model independence of tetramer properties, when few physical scales are fixed, was recently numerically established by our group $[11,12]$ through the analysis of observed correlations between tetramer observables by considering a renormalized zero-range (RZR) approach. These correlations were realized through appropriate scaling functions, from where one can verify that the RZR results are consistent with several other model calculations [13-16].

Despite the success of the model independence of the tetramer scaling functions, the experimental data for cesium atoms $[6,8,9]$ are shifted with respect to the correlation function relating the peaks of successive tetramer resonances for a zero-range force [11]. Among the possible effects to explain such a deviation from the results obtained with zero-range two-body interaction, it is natural to think in a way to introduce the correction due to a finite effective range in the model, as suggested in Ref. [17].

The experimental data for three-body cold-atom reactions show that Efimov resonances and recombination minima survive in the intermediate regime when the scattering length $a$ exceeds the characteristic interaction range by a relatively small factor [18]. But the positions are shifted away from the naive predictions based on the universal theory $[14,15]$ and finite range potentials have been used $[19,20]$ to address such issues. On the other hand, the quantitative reproduction of the experimental findings for the positions of the peaks of the tetramer resonances has not yet been addressed in detail.

Within our aim of clarifying the shift in the experimental data with respect to the zero-range predictions for the positions of the tetramer resonances at the dissociation point, we present an approach on how to introduce the effective range correction in lowest order in the zero-range four-body theory. We obtain the correction to the correlation of the positions of two successive resonant four-boson recombination peaks. The associated scaling function is not fully determined by the trimer properties and requires a four-boson scale [11]. Furthermore, we are showing by our calculations that the effective range correction is not completely parametrized by the trimer and tetramer short-range scales, as one could naively expect. The contribution of a nonzero effective range affects the observed scaling plots by moving the corresponding positions in relation to the zero-ranged ones (see, e.g., Refs. [19,21] in the case of trimers). Here it will be shown quantitatively how the effective range affects the scaling between negative scattering length ratios, corresponding to the dissociation positions of a trimer and related tetramers (for two nearby tetramer states, associated with a given trimer state).

Relevant to the understanding of the effective range contribution to the resonance positions, we should recall the valuable discussion by Chin et al. [10] on the role of broad (entrance-channel dominated) and narrow (closed-channel dominated) Feshbach resonances. As discussed, a Feshbach resonance strongly dominated by the entrance channel allows a description of a two-atom system in terms of a single-channel short-ranged model with a van der Waals tail. The broad $s$-wave Feshbach resonance of cesium [6,8,9], where tetramer dissociations at the four-body continuum threshold were observed, is dominated by an entrance channel. In this case, when the strength parameter is very large $\left(s_{\text {res }} \gg 1\right)$, the single-channel description is applicable [10] and consequently, the effective range should be found around the values obtained for van der Waals-like potentials, tuned to the scattering length of the resonance. Indeed, as we are going to detail in the present work, the estimated values we have obtained for the effective ranges, 
from the positions of tetramer resonances, are somewhat close to the corresponding effective range of that class of potentials. Within our approach to extract the effective range, we have considered broad Feshbach resonances appearing in ultracold gas of cesium atoms, leading to positive effective ranges. In contrast, narrow resonances, as discussed by Petrov [22], should lead to negative effective ranges. In the case of systems with narrow resonances and denser spectrums, we should also mention the recent work by Sørensen et al. [23].

The present work is organized as follows. In Sec. II the formalism for two, three, and four-boson systems is presented, where we address the effective range expansion of the twobody scattering amplitude, the subtracted Skorniakov and Ter-Martirosian (STM) equation, and Faddeev-Yakubovsky (FY) equations. In Sec. III the shifts in the position of four-body resonant loss peaks from the solution of the STM and FY equations with the effective range correction are given. In Sec. IV we performed an analysis of the positions of the four-boson resonance peaks for the cesium data close to wide Feshbach resonances. The effective range, shown to be compatible with experiments, is compared to the one obtained from van der Waals-like potentials for the $\mathrm{Cs}_{2}$ system. In Sec. V we present our conclusions and perspectives.

\section{FORMALISM FOR TWO, THREE, AND FOUR-BOSON SYSTEMS}

\section{A. Two-body scattering amplitude with effective range correction}

The $s$-wave two-boson scattering amplitude input to the subtracted forms of the trimer Skorniakov and Ter-Martirosian equation [24] and the tetramer coupled Faddeev-Yakubovsky equations [25] for negative energies $\epsilon$, with the on-shell momentum given by $k=i \sqrt{-\epsilon}$, can be written as

$$
\tau(\epsilon) \approx \frac{1 /\left(2 \pi^{2}\right)}{-\sqrt{-\epsilon} \pm \sqrt{-\epsilon_{2}}}\left[1+\frac{r_{0}}{2}\left(\sqrt{-\epsilon} \pm \sqrt{-\epsilon_{2}}\right)\right]
$$

in the lowest order of the effective range $r_{0}$. In the above, the pole of the scattering amplitude is fixed by the bound $(+)$ or virtual state $(-)$ energy, $k^{2}=\epsilon=-\left|\epsilon_{2}\right|$. The inverse of the scattering length, up to the same order, is

$$
\frac{1}{a}= \pm \sqrt{\left|\epsilon_{2}\right|}-\frac{r_{0}}{2}\left|\epsilon_{2}\right| \text {. }
$$

The two-body amplitude (1) provides a correction to the STM and FY equations in leading order in $r_{0}$, and as we verified, the subtracted forms of these equations are able to provide finite results dealing with the extra power of momentum in the amplitude.

In the following, we present some details on the formalism for the subtracted equations we have used together with our approach to find the effective range correction for the position of the tetramer resonances.

\section{B. Subtracted STM equation}

The Faddeev components for three-boson bound states for the zero-range potential can be rewritten in terms of spectator functions, which are solutions of a subtracted STM integral equation given by [24]

$$
\left|\mathcal{K}_{i j, k}\right\rangle=2 \tau\left(\epsilon_{i j, k}\right) \mathcal{G}_{i j ; i k}^{(3)}\left|\mathcal{K}_{i k, j}\right\rangle,
$$

where $\epsilon_{i j, k}$ is the $(i j)$ subsystem energy in the three-body system. Note that we are going to introduce the two-boson amplitude (1) carrying the effective range correction in (3). The subtraction of the free three-body resolvent regulates the STM equation and the projected operator $\mathcal{G}^{(3)}$ is

$$
\mathcal{G}_{i j ; i k}^{(3)}=\left\langle\chi_{i j}\left|\left(\left[E-H_{0}\right]^{-1}-\left[-\mu_{3}^{2}-H_{0}\right]^{-1}\right)\right| \chi_{i k}\right\rangle,
$$

where $H_{0}$ is the three-body free Hamiltonian and $-\mu_{3}^{2}$ is the subtraction energy scale. The form factor appearing in the two-body $\mathrm{T}$ matrix of the pair $(i j)$, in the relative momentum $\mathbf{p}_{i j}$ is given by $\left\langle\mathbf{p}_{i j} \mid \chi_{i j}\right\rangle=1$. The three-body regularization parameter $\mu_{3}$ keeps under control the Thomas collapse and determines the three-boson observables, and can be parametrized by one $s$-wave physical quantity (see, e.g., [26]). The subtracted STM equations were also used to calculate the position of triatomic continuum resonances for large negative scattering lengths [27], which were observed by the Innsbruck group [2] through a three-atom recombination peak in a cold cesium gas close to a Feshbach resonance.

\section{Subtracted FY equations}

The tetramer energies are found by solving the FY equations properly regulated in the limit of the zero-range interaction, which are written as a set of coupled subtracted integral equations $[11,12,25]$ given by

$$
\begin{gathered}
\left|\mathcal{K}_{i j, k}^{l}\right\rangle=2 \tau\left(\epsilon_{i j, k}^{l}\right)\left[\mathcal{G}_{i j ; i k}^{(3)}\left|\mathcal{K}_{i k, j}^{l}\right\rangle+\mathcal{G}_{i j ; i k}^{(4)}\left(\left|\mathcal{K}_{i k, l}^{j}\right\rangle+\left|\mathcal{H}_{i k, j l}\right\rangle\right)\right], \\
\left|\mathcal{H}_{i j, k l}\right\rangle=\tau\left(\epsilon_{i j, k l}\right) \mathcal{G}_{i j ; k l}^{(4)}\left[2\left|\mathcal{K}_{k l, i}^{j}\right\rangle+\left|\mathcal{H}_{k l, i j}\right\rangle\right]
\end{gathered}
$$

The subtractions in the kernel come at the level of the free four-body propagators in Eqs. (5) and (6), which are used to regulate the FY equations for the contact potential. The two-boson scattering amplitude (1), which is introduced in the FY equations above, carries the effective range correction in the calculations that are presented in the next section.

The projected four-body free resolvent $\mathcal{G}^{(4)}$ is subtracted at an energy $-\mu_{4}^{2}$ :

$$
\mathcal{G}_{i j ; i k}^{(4)}=\left\langle\chi_{i j}\left|\left(\left[E-H_{0}\right]^{-1}-\left[-\mu_{4}^{2}-H_{0}\right]^{-1}\right)\right| \chi_{i k}\right\rangle,
$$

where $H_{0}$ is now the free four-body Hamiltonian. Notice that when introducing $\mathcal{G}_{i j ; i k}^{(3)}$ [Eq. (4)] in the FY equations, the free three-body Hamiltonian should be substituted by the four-body one. The energy of the two-body subsystem $(i j)$, appearing as arguments of the two-boson scattering amplitude in Eqs. (5) and (6), are $\epsilon_{i j, k}^{l}$ and $\epsilon_{i j, k l}$, associated with a virtual pair in the $3+1$ partition and in the $2+2$ partition, respectively.

The subtraction scale $-\mu_{3}^{2}$ in $\mathcal{G}_{i j ; i k}^{(3)}$, the right-hand side of Eq. (5), fix the trimer properties consistently with the STM equation but independently on the scale $\mu_{4}$. Looking closer to this term and taking into account the left-hand side, the position of the trimer pole is guaranteed to be the same as the bound state energy obtained by solving the subtracted STM equation. The other terms in the FY equations, in (5) and (6), are regularized with an independent subtraction scale $-\mu_{4}^{2}$. 
This method ensures that the three-body subtraction scale fixes the three-body properties consistently with the subtracted STM equation, whereas the regularization parameter $\mu_{4}$ fixes the four-body observables [25].

\section{POSITION OF FOUR-BODY RESONANT LOSS PEAKS}

The four-atom recombination resonates when the tetramer has zero energy and is placed at the four-body continuum threshold. In cold atom traps the resonance is reached by tuning the negative scattering length close to a Feshbach resonance (see, e.g., [18]). The theoretical description of the scaling function, which correlates the values of the negative scattering lengths where successive tetramers reach the continuum, introduced in [11], is now extended to include the effective range, as

$$
a_{N_{3}, N+1}^{T}=a_{N_{3}}^{-} \mathcal{A}\left(\frac{a_{N_{3}, N}^{T}}{a_{N_{3}}^{-}}, \frac{r_{0}}{a_{N_{3}}^{-}}\right),
$$

where $a_{N_{3}}^{-}$is the position of the peak of the three-atom resonant recombination for $a<0$, and $a_{N_{3}, N}^{T}$ is the scattering length for which the excited $N$ th tetramer dissociates and meets the four-body continuum. The solutions of the subtracted STM (3) and FY equations (5) and (6) depend only on $a, r_{0}$, and on the three- and four-body scale parameters. Then for the zero energy tetramer, the value of the negative scattering length depends only on $r_{0}$ and the short-range three- and four-body momentum scales at the subtraction points. The three-body scale can be parametrized by $a_{N_{3}}^{-}$, while the four-body one by $a_{N_{3}, N}^{T}$ in the determination of $a_{N_{3}, N+1}^{T}$. In this way (8) can be built. Furthermore, the scaling function $\mathcal{A}$ is calculated with the two-boson scattering amplitude (1) and the solutions of the FY equations, as well as of the STM equation, expanded up to first order in $r_{0}$.

The dimensionless function $\mathcal{A}$ is determined by solving the subtracted STM [24] and FY [25] equations considering the expansion of the atom-atom $s$-wave amplitude up to order $k^{2}$ as given by (1) and the corresponding relation between the scattering length and virtual state energy (2). The solutions of the subtracted FY equations depend only on $a, r_{0}$, and on the three- and four-body scale parameters. Then, for the zero energy tetramer, the value of the negative scattering length depends only on $r_{0}$ and the short-range three- and four-body momentum scales at the subtraction points. The three-body scale can be parametrized by $a_{N_{3}}^{-}$, when solving the STM equation with $r_{0}$ included, while the four-body scale is correlated to $a_{N_{3}, N}^{T}$ in the determination of $a_{N_{3}, N+1}^{T}$. Then the scaling function (8) is built without reference to the scale ratio by eliminating this dependence in the ratio $a_{N_{3}, 2}^{T} / a_{N_{3}}^{-}$in terms of $a_{N_{3}, 1}^{T} / a_{N_{3}}^{-}$.

In our calculations we have only obtained results for tetramers below the ground-state trimer, which should be enough for our study, as the values of the scattering length are much larger than the short-range length scales corresponding to the three- and four-body subtraction points. The scaling function $\mathcal{A}\left(a_{1,1}^{T} / a_{1}^{-}, r_{0} / a_{1}^{-}\right)$, which provides the correction to the position of the four-atom resonance, is shown in the threedimensional (3D) plot of Fig. 1. We have presented results up to $r_{0} / a_{1}^{-} \sim 0.5$, for $0.40 \leqslant a_{1,1}^{T} / a_{1}^{-} \leqslant 0.51$, considering

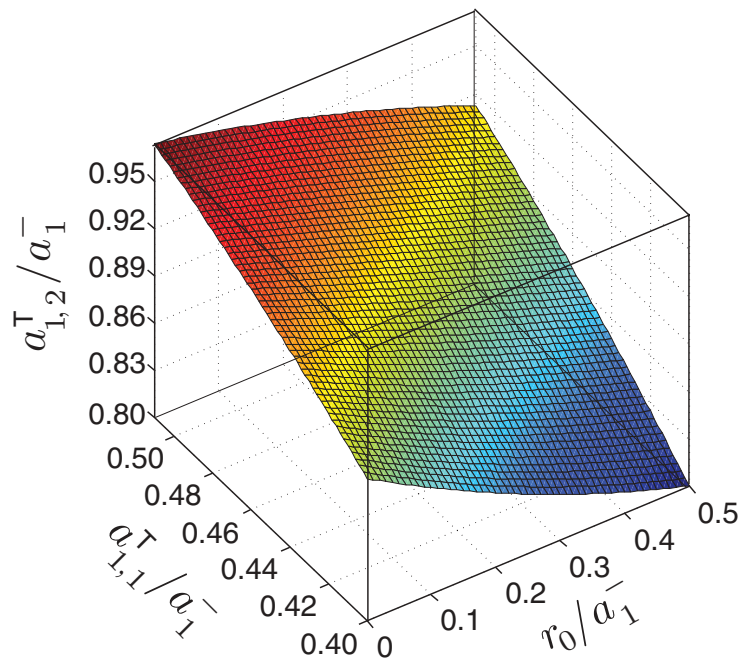

FIG. 1. (Color online) The scaling function $\mathcal{A}$ expressing the dependence of $a_{1,2}^{T} / a_{1}^{-}$in $a_{1,1}^{T} / a_{1}^{-}$and $r_{0} / a_{1}^{-}$. Calculations are performed in a leading-order expansion of the solutions of the FY equations with the two-body amplitude (1). (See text for further explanation.)

the relevant region where recombination data for cesium have been measured. In this case, $r_{0} \sim a_{1,1}^{T}$, and for the validity of the expansion in the effective range one should have $r_{0} \ll a_{1,1}^{T}$, and the expansion of the scattering length (2) is questionable. Fortunately, we found that the coefficients for the effective range correction are fairly small, allowing extension of the validity of the expansion to the region where data are found.

Within the region of the plot given in Fig. 1, the surface can be parametrized by

$$
\frac{a_{1,2}^{T}}{a_{1}^{-}}=\mathcal{A}(x, y)=\sum_{0 \leqslant m+n \leqslant 2} c_{m n}(x-0.45)^{m} y^{n},
$$

where $x \equiv a_{1,1}^{T} / a_{1}^{-}, y \equiv r_{0} /\left|a_{1}^{-}\right|$and $(m, n) \geqslant 0$. The coefficients are given in Table I.

Although the expansion (9) contains nonlinear terms in $r_{0}$, it is built in linear order in the solutions of the subtracted STM and FY equations. The second-order terms are induced by the curvature of $\mathcal{A}(x, y)$ in $x$ and $y$. In our case the calculation is performed for the ground-state trimer and two successive tetramer states.

The coefficients for the effective range correction are expected to be smaller than the ones associated with the variation of $a_{1,1}^{T} / a_{1}^{-}$. Indeed, the expansion around $a_{1,1}^{T} / a_{1}^{-}=$ 0.45 shows that the linear coefficient for $r_{0} / a_{1}^{-}$correction gives $c_{01} / c_{00}=-0.15$, while $c_{10} / c_{00}=0.78$ is 5 times larger. The amazing smallness of $c_{01}$ and $c_{02}$ with respect to the other coefficients reflects that a fraction of the effect from the effective range is absorbed by the variation of the short-range four-body scale, but not all. Given that, it is quite obvious that

TABLE I. Coefficients for the parametrization (9).

\begin{tabular}{cccccc}
\hline \hline$c_{00}$ & $c_{10}$ & $c_{01}$ & $c_{11}$ & $c_{20}$ & $c_{02}$ \\
\hline 0.932 & 0.724 & -0.144 & 0.347 & -0.645 & 0.001 \\
\hline \hline
\end{tabular}




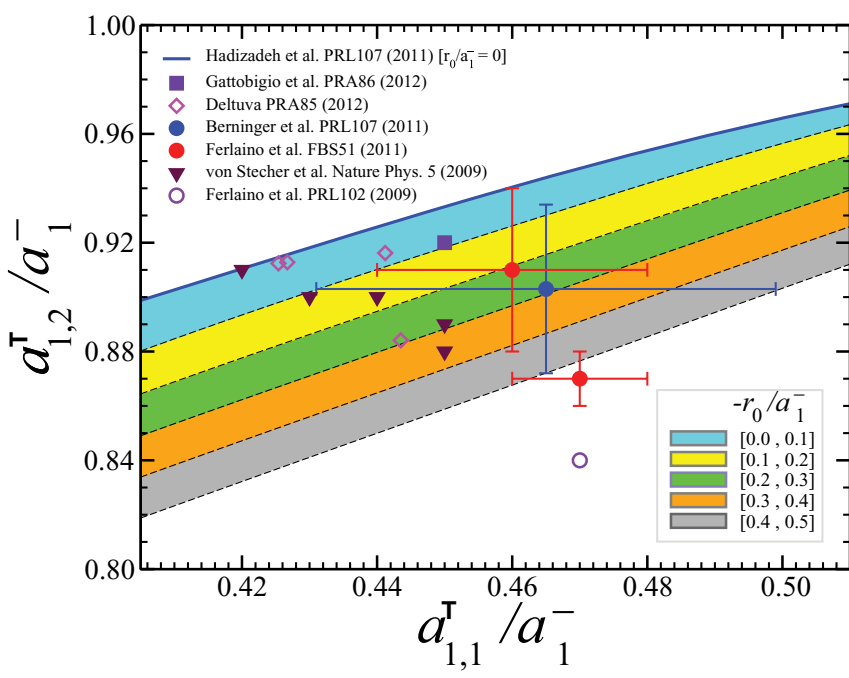

FIG. 2. (Color online) Lower-order effective range correction for the scaling plot $a_{1,2}^{T} / a_{1}^{-}=\mathcal{A}\left(a_{1,1}^{T} / a_{1}^{-}, r_{0} / a_{1}^{-}\right)$for the correlation between the positions of the peaks for successive tetramer resonances at the continuum threshold, compared to other theoretical results and experimental data. We present our results for different $r_{0}$ intervals (the upper solid line is from [11]) compared to a few recent calculations as indicated inside the frame [Refs. [13] (triangles), [16] (diamonds), and [30] (square)]. The experimental data of Refs. [6,8,9] are also indicated inside the figure. The lowest band (gray) corresponds to $r_{0}$ for the van der Waals-like potential for cesium atoms with values within the interval $0.4 \lesssim-r_{0} / a_{1}^{-} \lesssim 0.5$ for a reference value of $a_{1}^{-} \simeq-9 R_{\mathrm{vdW}}$ (see Table II).

the correction coefficients for the effective range contribution, shown in Table I, should be somewhat smaller than the coefficients associated with the expansion parameter $a_{1,1}^{T} / a_{1}^{-}$.

The negative value of $c_{01}$ decreases the function $\mathcal{A}$ for positive effective ranges, which suggests that the region where the two-body amplitude (1) increases with respect to the zerorange one, for $a<0$ and positive effective ranges, is important for the tetramers. In this case, larger variations of the scattering length toward negative values, compared to the trimer value of $a_{1}^{-}$, are required to tune the tetramers to zero energy.

Two theoretical results are used for comparison with the results of the scaling function $\mathcal{A}$, using the plot of Fig. 2, the first one by Deltuva [16] $(0.4435,0.8841)$, which quotes $r_{0} /\left|a_{1}^{-}\right|=0.33$ [28] compared to 0.29 extracted from the plot, and another one by von Stecher et al. [13], (0.45, 0.88), with $r_{0} /\left|a_{1}^{-}\right|=0.38$ [29] compared to 0.36 obtained from the plot. This comparison with completely different models, the separable potential model [16] and local Gaussian potential
[13], gives us confidence on the universality and utility of the function $\mathcal{A}$ to analyze the data. This comparison suggests a lower bound of $\left|r_{0} / a_{1}^{-}\right|>0.02$ in our extraction method.

In Fig. 2, the two-dimensional (2D) plot of our results for the surface $\mathcal{A}(x, y)$, corresponding to the scaling function (8), is shown. The bands in the plot give the shift of the scaling function due to the finite values of $r_{0} /\left|a_{N_{3}}^{-}\right|$in the intervals depicted in the figure. For our reference we also plot the theoretical results from $[13,16]$, and the experimental values from Refs. [6,8,9]. The data from Ref. [7] have large error bars and suggest $r_{0}$ ranging from negative to positive values.

\section{ANALYSIS OF THE CESIUM DATA}

The effective range extracted from the experimental data is shown in Table II. They were obtained just by inspection of Fig. 2, by comparing the experimental values with the scaling curve including the effective range correction. The shift of the data yields values of $r_{0} / a_{1}^{-}$larger than the lower bound for the theoretical extraction. We did not present results for [7] as it has larger errors. We just notice that the effective ranges from these data vary from negative to positive values. The extraction of $r_{0}$ from the data for cesium atoms close to Feshbach resonances are within consistent errors. The extracted values of $r_{0}$ vary from 2 to 5 times the van der Waals length $\left(R_{\mathrm{vdW}}\right)$, which for the $\mathrm{Cs}_{2}$ system is $R_{\mathrm{vdW}}^{C s_{2}}=101.0 a_{0}$ [10].

The effective range of potentials with van der Waals tail $-C_{6} / r^{6}$ at large distances has the approximate formula given by [31-33]

$r_{0}=\frac{2}{3} \frac{\Gamma(1 / 4)^{4}}{(2 \pi)^{2}} \bar{a} \vartheta \quad$ and $\quad \vartheta=\left(\frac{\bar{a}}{a}\right)^{2}+\left(\frac{\bar{a}}{a}-1\right)^{2}$,

where $\bar{a}=2 \pi / \Gamma(1 / 4)^{2} R_{\mathrm{vdW}} \simeq 0.955978 R_{\mathrm{vdW}}$ is the average scattering length of van der Waals potentials [33], with the van der Waals length $R_{\mathrm{vdW}}=\frac{1}{2}\left(m C_{6} / \hbar\right)^{1 / 4}$. Considering the van der Waals length one gets that $\bar{a}=96.5 a_{0}$ and

$$
r_{0} \simeq 2.7894 R_{\mathrm{vdW}} \vartheta=2.9179 \bar{a} \vartheta \simeq 281.7 \vartheta a_{0} .
$$

In the actual cold cesium experiments listed in Table II, the factor $\vartheta$ varies from 1.26 (for the second tetramer resonance) to 1.55 (for the first tetramer resonance), which gives $r_{0}$ within 3.5-4.3 $R_{\mathrm{vdW}}$. The corresponding interval of $0.4 \lesssim r_{0} /\left|a_{1}^{-}\right| \lesssim$ 0.5 for a reference value of $a_{1}^{-} \simeq-9 R_{\mathrm{vdW}}$ can be identified with the lowest band (gray) shown in Fig. 2.

The estimated values of $r_{0}$ extracted from the shift of the data with respect to the zero-range calculations, shown in Fig. 2, are given in Table II. The values for the effective range are found between $2 R_{\mathrm{vdW}}$ and $\sim 5 R_{\mathrm{vdW}}$, while Eq. (11)

TABLE II. Extracted effective ranges from the shift of the experimental peaks of the four-atom losses for a gas of cold cesium atoms close to a Feshbach resonance using the scaling plot shown in Fig. 2. The errors in the extracted values of $r_{0}$ are estimated from the figure as well.

\begin{tabular}{cllcc}
\hline \hline Ref. & $a_{1,1}^{T} / a_{1}^{-}$ & $a_{1,2}^{T} / a_{1}^{-}$ & $a_{1}^{-}$[units of $R_{\text {vdw }}$ ] & $r_{0}$ [units of $R_{\text {vdw }}$ ] \\
\hline$[6]$ & 0.47 & 0.84 & $-8.7(1)$ & $>5$ \\
{$[8]$} & $0.465(34)$ & $0.903(31)$ & $-9.54(28)$ & $2.5 \pm 1.7$ \\
{$[9]$} & $0.47(1)$ & $0.87(1)$ & -8.71 & $4.8 \pm 1.0$ \\
{$[9]$} & $0.46(2)$ & $0.91(3)$ & -9.64 & $2 \pm 2$ \\
\hline \hline
\end{tabular}


applied to the set of cesium data suggests the interval $3.5-4.3 R_{\mathrm{vdW}}$ represented by the lowest (gray) band in the figure. The present experimental errors overlap the cesium data, as one can verify in Fig. 2. By performing the errorweighted average of the extracted effective range values from the data given in Refs. [8,9] we obtain $3.9 \pm 0.8 R_{\mathrm{vdW}}$. In particular, the data set $\{0.47(1), 0.87(1)\}$ provides an effective range of about $4.8 R_{\mathrm{vdW}}$, which within $1 \sigma$ is consistent with the values of the van der Waals potential. The data set $\{0.47,0.84\}$ [6] with no quoted errors suggests a large value of $r_{0}>5 R_{\mathrm{vdW}}$.

It is noticeable that the dissociation points of the cesium ground-state Efimov trimers for negative scattering lengths are found in a narrow band [8], independent of which Feshbach resonance the system is tuned (see Table II). Theoretical works [23,34-37] addressed the interesting issue of the physical mechanism for the dominance of the two-body properties on the position of the first Efimov resonance for $a<0$, when the trimer meets the continuum. The results in Table II show a range of values for $r_{0}$ extracted from the tetramer and trimer resonances, which within errors (excepting one data set) are consistent with the effective range values from the van der Waals-type potential. The numerical analysis of Wang et al. [34] has shown that two-body interactions, which suppress efficiently the wave function for separation distances less than $r_{0}$, have $a_{1}^{-} \sim-9 R_{\mathrm{vdW}}$, determined by two-body properties, with this class of systems closely related to entrance-channel-dominated Feshbach resonances. The dominance of the single-channel potential with the van der Waals tail in the three-cesium reactions, with suppression of the short-distance wave function, put forward the narrow band where the trimer dissociation position is found. It also suggests that the effective range, seen through the shift of the position of the four-atom recombination peak with respect to the zero-range results, should be given by the van der Waals tail of the potential, as well.

It is worthwhile to address the validity of the correction due the effective range, considering the main results presented in Fig. 2 and Table II. In view of the expansion given in Eq. (1), which at a first glance would be doubtful, when $r_{0} /\left|a_{1}^{-}\right| \sim a_{12}^{T} / a_{1}^{-}$, we found consistency between the present calculations with the results obtained with short-range separable [28] and local [29] interactions. The leading coefficient for the expansion in $r_{0} /\left|a_{1}^{-}\right|$is one fifth of the corresponding coefficient for the dependence of the scaling function in $a_{11}^{T} / a_{1}^{-}$ (see Table I), which shows that the extracted values of $r_{0}$ using the scaling plot come from small corrections to the zero-range scaling function. Therefore, as observed in Fig. 2, the agreement of the expansion we have considered can be extended also to cases near $a_{12}^{T} / a_{1}^{-} \sim r_{0} /\left|a_{1}^{-}\right|$.

\section{CONCLUSION AND OUTLOOK}

In this work we present an approach to extract the effective range of a given atomic system from the shifts in the four-body recombination peaks, at the tetramer-dissociation threshold, with respect to the zero-range results. We solved the trimer and tetramer subtracted zero-range integral equations with twobody amplitudes carrying the effective range in lowest order. Besides the trimer and tetramer subtracting scales, no further parameters are introduced in the calculations. The correlation between the negative scattering lengths where successive tetramers dissociate at the four-body continuum is extended to include the correction due to the effective range, which is model independent when checked against different short-range potential model calculations. We found that the effective range correction of the on-shell two-body amplitude in the calculation of the trimer and tetramer-dissociation points, when presented as a correlation plot of the successive positions of the tetramer resonances, is not completely parametrized by the trimer and tetramer short-range scales, as one could naively expect. This unexpected property turned out to be essential to single out the effective range from the shift in the positions of the four-atom recombination peaks with respect to the zero-range theory.

We applied our proposal to the cesium data measured at broad $s$-wave Feshbach resonances $[6,8,9]$. In our analysis we considered the shifts of the observed recombination peaks at the tetramer-dissociation positions with respect to the zero-range results. The effective ranges were found within the interval $2 R_{\mathrm{vdW}} \lesssim r_{0} \lesssim 5 R_{\mathrm{vdW}}$, with a reference value of $3.9 \pm 0.8 R_{\mathrm{vdW}}$, obtained by the error-weighted average of the $r_{0} \mathrm{~s}$ extracted from the data of Refs. [8,9]. These values are consistent with the van der Waals potential tail for the broad $s$-wave Feshbach resonances of the $\mathrm{Cs}_{2}$ system, providing $r_{0}$ within the interval of $3.5-4.3 R_{\mathrm{vdW}}$ for the scattering lengths where the resonances were found. Our results provide strong evidence regarding the prevalence of entrance-channel dominance in the physics of the universal tetramers formed with broad Feshbach resonances.

The question regarding how the effective range is sensitive to Feshbach resonance manipulations in the scattering length as suggested by (11) has to be considered. Once improved experimental data are available, it will be possible to verify in more detail how Feshbach resonances from different setups affect the $r_{0}$ values extracted from the trimer and tetramer-dissociation points. The present analysis, based on the universality of effective range correction to the position of tetramer resonances, can be generally applied to any other cold-atom bosonic system close to Feshbach resonances.

\section{ACKNOWLEDGMENTS}

We thank A. Deltuva and J. von Stecher for sending us some of their effective range results. T.F. and L.T. also appreciate clarifying discussion with P. Naidon and S. Endo. Our thanks also to Fundação de Amparo a Pesquisa do Estado de São Paulo and Conselho Nacional de Desenvolvimento Científico e Tecnológico for partial support.
[1] V. Efimov, Phys. Lett. B 33, 563 (1970); Sov. J. Nucl. Phys. 12, 589 (1971).

[2] T. Kraemer, M. Mark, P. Waldburger, J. G. Danzl, C. Chin, B. Engeser, A. D. Lange, K. Pilch, A. Jaakkola,
H.-C. Nägerl, and R. Grimm, Nature (London) 440, 315 (2006).

[3] S. Knoop, F. Ferlaino, M. Mark, M. Berninger, H. Schöbel, H.-C. Nägerl, and R. Grimm, Nat. Phys. 5, 227 (2009). 
[4] M. Zaccanti, B. Deissler, C. D’Errico, M. Fattori, M. JonaLasinio, S. Müller, G. Roati, M. Inguscio, and G. Modugno, Nat. Phys. 5, 586 (2009).

[5] G. Barontini, C. Weber, F. Rabatti, J. Catani, G. Thalhammer, M. Inguscio, and F. Minardi, Phys. Rev. Lett. 103, 043201 (2009).

[6] F. Ferlaino, S. Knoop, M. Berninger, W. Harm, J. P. D’Incao, H.-C. Nägerl, and R. Grimm, Phys. Rev. Lett. 102, 140401 (2009).

[7] S. E. Pollack, D. Dries, and R. G. Hulet, Science 326, 1683 (2009).

[8] M. Berninger, A. Zenesini, B. Huang, W. Harm, H.-C. Nagerl, F. Ferlaino, R. Grimm, P. S. Julienne, and J. M. Hutson, Phys. Rev. Lett. 107, 120401 (2011).

[9] F. Ferlaino, A. Zenesini, M. Berninger, B. Huang, H.-C. Nägerl, and R. Grimm, Few-Body Syst. 51, 113 (2011).

[10] C. Chin, R. Grimm, P. Julienne, and E. Tiesinga, Rev. Mod. Phys. 82, 1225 (2010).

[11] M. R. Hadizadeh, M. T. Yamashita, L. Tomio, A. Delfino, and T. Frederico, Phys. Rev. Lett. 107, 135304 (2011).

[12] M. R. Hadizadeh, M. T. Yamashita, L. Tomio, A. Delfino, and T. Frederico, Phys. Rev. A 85, 023610 (2012).

[13] J. von Stecher, J. P. D'Incao, and C. H. Greene, Nat. Phys. 5, 417 (2009).

[14] H.-W. Hammer, T. A. Lahde, and L. Platter, Phys. Rev. A 75, 032715 (2007).

[15] L. Platter, C. Ji, and D. R. Phillips, Phys. Rev. A 79, 022702 (2009).

[16] A. Deltuva, Phys. Rev. A 85, 012708 (2012).

[17] T. Frederico, L. Tomio, A. Delfino, M. R. Hadizadeh, and M. T. Yamashita, Few-Body Syst. 51, 87 (2011).

[18] F. Ferlaino and R. Grimm, Physics 3, 9 (2010).
[19] M. Thøgersen, D. V. Fedorov, and A. S. Jensen, Phys. Rev. A 78, 020501(R) (2008).

[20] M. Jona-Lasinio and L. Pricoupenko, Phys. Rev. Lett. 104, 023201 (2010).

[21] T. Frederico, L. Tomio, A. Delfino, and A. E. A. Amorim, Phys. Rev. A 60, R9 (1999).

[22] D. S. Petrov, Phys. Rev. Lett. 93, 143201 (2004).

[23] P. K. Sørensen, D. V. Fedorov, A. S. Jensen, and N. T. Zinner, Phys. Rev. A 86, 052516 (2012).

[24] S. K. Adhikari, T. Frederico, and I. D. Goldman, Phys. Rev. Lett. 74, 487 (1995)

[25] M. T. Yamashita, L. Tomio, A. Delfino, and T. Frederico, Europhys. Lett. 75, 555 (2006).

[26] M. T. Yamashita, T. Frederico, A. Delfino, and L. Tomio, Phys. Rev. A 66, 052702 (2002).

[27] F. Bringas, M. T. Yamashita, and T. Frederico, Phys. Rev. A 69, 040702(R) (2004).

[28] A. Deltuva (private communication).

[29] J. von Stecher (private communication).

[30] M. Gattobigio, A. Kievsky, and M. Viviani, Phys. Rev. A 86, 042513 (2012).

[31] G. F. Gribakin and V. V. Flambaum, Phys. Rev. A 48, 546 (1993).

[32] B. Gao, J. Phys. B 37, 4273 (2004).

[33] C. Chin, V. Vuletić, A. J. Kerman, S. Chu, E. Tiesinga, P. J. Leo, and C. J. Williams, Phys. Rev. A 70, 032701 (2004).

[34] J. Wang, J. P. D’Incao, B. D. Esry, and C. H. Greene, Phys. Rev. Lett. 108, 263001 (2012).

[35] P. Naidon, E. Hiyama, and M. Ueda, Phys. Rev. A 86, 012502 (2012).

[36] P. Naidon, S. Endo, and M. Ueda, arXiv:1208.3912v1.

[37] R. Schmidt, S. P. Rath, and W. Zwerger, Eur. Phys. J. B 85, 386 (2012). 